\title{
Determining of Proxy and Self-report Agreement on the Stroke and Aphasia Quality of Life Scale SAQOL-39/TR
}

\author{
Eda lyigün* and Bülent Toğram \\ Department of Speech and Language Therapy, Faculty of Health Sciences, Anadolu University, Turkey
}

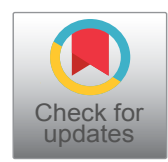

*Corresponding author: Eda lyigün, Department of Speech and Language Therapy, Faculty of Health Sciences, Anadolu University, Yunus Emre Campus, 26210, Tepebaşi, Eskişehir, Turkey, Tel: +90-554-774-70-49

\begin{abstract}
Health-related quality of life measures are increasingly used to evaluate after stroke conditions. However, after-stroke people with severe aphasia may not able to self-report on such scales due to the severity of their communication impairment and their proxies report on behalf of them.

This research aims to determine the level of agreement between people with aphasia and their proxies on the Turkish Stroke and Aphasia Quality of Life Scale-39 (SAQOL-39TR). For this purpose, 32 people with aphasia (whose auditory comprehension is good according to Aphasia Language Evaluation Test (ADD)) and their proxies in total 64 people were asked to report onSAQOL-39-TR.

Results show that proxy and self-agreement on theSAQOL39-TR for the total of the scale is excellent (ICC $=0.8$ ); for physical (ICC $=0.7)$, communication $(I C C=0.6)$, psycho-social (ICC $=0.6)$, and energy $(I C C=0.6)$ subtests, fair to a good degree of agreement is observed. No correlation between the SAQOL-39-TR, and subtests scores, age and gender variables were found for people with aphasia. However, there is a positive correlation between ADD's (Aphasia Language Evaluation Test) auditory comprehension score and communication subtest score of SAQOL-39-TR $(r=0.4$; $p<0.05)$.

The study reveals that there is a correlation between Proxies' Caregiver Well-Being Scale scores and SAQOL-39-TR scores $(r=0.446, p<0.05)$; communication subtest scores $(r=0.504, p<0.01)$; and psycho-social subtest scores $(r=$ $0.483, p<0.01)$.
\end{abstract}

\section{Keywords}

Aphasia, Proxy, Quality of life, Stroke

\section{Introduction}

Quality of life is defined as 'individuals' perception of their position in life in the context of culture and value systems in which they live and in relation to their goals, expectations, standards and concerns" [1]. There are different definitions for quality of life; however, it can refer to the difference between the expectations of people and their fulfillness [2]. Quality of life-related to health is the perception of people's health and themselves [3].

Health-related quality of life (HRQL) measures are increasingly used to evaluate health interventions. These are especially useful for people with long-term health problems [4]. Stroke is one of the conditions which affects health-related quality of life $[5,6]$. Previous research found that patient's post-stroke quality of life deteriorated due to physical disabilities, depression, and lack of social support [7]. Stroke is the most common cause of aphasia [8] and has long-term effects on the living. Aphasia has a considerable effect on the health-related life qualities of people experiencing stroke [9]. Hilari, Needle, and Harrison indicated that the degree of emotional stress, decrease in activity, accompanying communicative disorders are the predictors of the decrease within the quality of life of individuals with aphasia [10]. There are also other studies identified similar factors (such as, severity of aphasia and communication impairment; social and economic status, education level [11] as well as mood disorders [12] that affect the health-related quality of life. Bullier, et al. also point out that severity of aphasia, mood disorders and functional 
limitations are associated with poor quality of life [13]. Measuring the quality of life provides opportunities for clinicians to measure and understand the impacts of the disease on the patients' life [14]. They are also important for the inclusion of patient's views on the decision process in the clinic [15]. It may help clinicians provide better rehabilitation service, which would better contribute to increasing their life qualities and well-being status of patients [16].

As far as the quality of life for people with aphasia is concerned, four areas such as physical, functional, psychological and social health are taken into account [10]. These four areas should be included during the evaluation of the quality of life for people with aphasia. Physical health includes the symptoms as indicators of disease. Functional health includes self-care, and physical activity. Emotional status, the general perception of the patient of his/her health, his/her life satisfaction and happiness can be taken into account under psychological health. Lastly, social health includes social connections and interactions [17].

Studies on the quality of life of individuals with aphasia usually target individuals with mild and moderate severity of aphasia $[18,19]$. Nevertheless, there are individuals with poor auditory comprehension as a result of their aphasia which affects their ability to fill out the health-related quality of life scales in a negative way [20]. Therefore, these individuals are not able to participate in the clinical evaluations, service assessments, and post-stroke quality of life studies [21]. To prevent this, health related quality of life scales are filled out not by the individuals with aphasia but their proxies on their behalf by considering their emotions and views. Proxies are the ones who take care of the people with aphasia (e.g., wife, sister, brother, daughter, son, or the caregiver of the person with aphasia).

Mayou and Bryant emphasized the importance of including the patient's view in the clinical decision-making process [15]. It indicated that such an inclusion would help to provide rehabilitation services better in favor of improving the quality of lives and well-being of individuals [16]. However, individuals with aphasia whose auditory comprehension is low and who cannot fill out the quality-of-life scales are not able to participate in the studies measuring the quality of life $[20,21]$. Therefore, it can be concluded that in quality-of-life researches and rehabilitation services where information on the quality of life of individuals with aphasia cannot be obtained, such information can be obtained through their proxies.

The agreement level of the answers for the health-related quality-of-life scale between the proxies and the individuals with aphasia increases throughout the time that passes after the stroke $[5,22]$. In addition to that, it was observed that proxies usually got fewer scores of health-related quality-of-life than self-reports of in- dividuals with aphasia, but their scores became more harmonized in more observable areas such as physical conditions [22]. Cruice, et al. found significant differences between general health-related life evaluations of individuals with aphasia and their proxies [21]. Hilari, Owen, and Farrelly investigated the same questions by using SAQOL-39 [23]. Their study revealed that although there were significant differences between individuals with aphasia and proxies thereof, the agreement was at mild-moderate level (0.2-0.5) and the correlation of the scores of proxies and self-reports of individuals with aphasia was high in total scores, physical, psychological and communication areas (ICC $=0.7-0.8$ ). Although these studies significantly contribute to the literature, they need to be repeated for different societies in order to see whether aphasia and proxies' agreement on the scores of SAQOL-39 changes according to cultural differences. People's pattern of taking care of their parents who are in need or their defining way of the disease is changing from culture to culture. In Turkish culture, people generally feel responsible for the care of their parents who is in need as the family ties in Turkey are seen very important. This responsibility put the weight of feelings on the shoulders of the people, too [24,25]. People from the Asia and east part of world include the stress, worry, extra burden as they have self-sacrificed tendency [24]. This research is conducted to see whether the cultural difference of Turkish people effects the agreement of self-report and proxies' responses on quality-of-life scales, or not.

Some studies are showing that the psychological well-being of proxies has an impact on the agreement reports of proxies and individuals with aphasia [26]. Contrary to these, Knapp and Hewison found that caregiver's well-being scores have no impact on the agreement of the health-related quality of life reports of proxies and those of individuals with aphasia [27]. Hilari, et al. found that caregiver's well-being has no impact on that agreement as a variable [23]. However, further research is needed on that variable. Ignatiou, et al. examined the agreement of the health-related quality of life reports of proxies and those of individuals with aphasia by adapting the SAQOL-39 scale for Greece, it is known as SAQOL-39g [28]. Results of this study were in parallel to those of Hilari, et al. [23]. In this study, we expected to replicate the findings of previous research obtained from the people with aphasia and their proxies in Turkey, too. It would be beneficial for the literature to what extent culture and language affect the agreement results between the reports given by individuals with aphasia and by their proxies to SAQOL-39.

The general purpose of this study is to examine the agreement between the reports given by individuals with aphasia and their proxies to SAQOL-39-TR. Differences and relations between the perceptions of individuals with aphasia and their proxies on health-related 
quality of life were examined by analyzing the impacts of certain variables such as age, gender, caregiver's well-being, which are considered to affect the degree of consistency.

\section{Method}

\section{Participants}

A total of 64 people, 32 individuals with aphasia and 32 proxies of individuals with aphasia, participated in our study. Demographic features of individuals with aphasia and their proxies were provided in Table 1.

\section{Inclusion criteria of participants}

Participants of the study were determined by applying criterion-referenced sampling method. Therefore, individuals with aphasia possessing the following criteria participated in this study: Having Turkish as the native language; being at an age older than 18; having a loss of speech for more than 4 months after onset of stroke; not having any pre-stroke cognitive or mental health problems; scoring at least 33 from the auditory comprehension subtest of Aphasia Language Evaluation Test (ADD) [29] and being literate before the stroke. In other words, the participants at least graduated from primary school. Criteria for proxies of individuals with aphasia to participate in this study were: Having Turkish as the native language; being at an age older than 18; contacting the individual with aphasia on a daily basis, not having any cognitive or mental health problem; and being literate. Participants meeting the aforementioned criteria were accepted into our study. The study was approved by the Anadolu University Ethics Committee (Protocol number: 8906) and participants gave their written informed consent conforming the study protocol before the initiation of the study.

\section{Data collection tools}

The Stroke and Aphasia Life Quality-39 Scale-TR and Caregiver Well-Being are used in this study. The test

Table 1: Demographic features of people with aphasia and their proxies.

\begin{tabular}{|c|c|c|}
\hline Variables & People with Aphasia n (\%) & Proxies of people with Aphasia $n(\%)$ \\
\hline \multicolumn{3}{|l|}{ Gender } \\
\hline Female & $12(37.5 \%)$ & $23(72 \%)$ \\
\hline Male & $20(62.5 \%)$ & $9(28 \%)$ \\
\hline \multicolumn{3}{|l|}{ Age } \\
\hline Mean & 57.4 (SS:12.1) & 50 (SS:12.8) \\
\hline Range & $19-73$ & $18-67$ \\
\hline \multicolumn{3}{|l|}{ Marital Status } \\
\hline Married & $31(97 \%)$ & \\
\hline Single & $1(3 \%)$ & \\
\hline $\begin{array}{l}\text { ADD Auditory Comprehension } \\
\text { Score } \\
\text { Mean } \\
\text { Range }\end{array}$ & $\begin{array}{l}50.2(\mathrm{SS}: 12.1) \\
32-66\end{array}$ & \\
\hline \multicolumn{3}{|l|}{ Degree of Relativity } \\
\hline Spouse & & $19(59.4 \%)$ \\
\hline Daughter/Son & & $8(25 \%)$ \\
\hline Sibling & & $2(6.3 \%)$ \\
\hline Care giver & & $1(3.1 \%)$ \\
\hline Other proxy & & $2(6.3 \%)$ \\
\hline $\begin{array}{l}\text { Caregiver Well-Being } \text { Scale } \\
\text { scores }\end{array}$ & & \\
\hline Mean & & 152 \\
\hline Range & & $103-206$ \\
\hline \multicolumn{3}{|l|}{ Basic Needs Subtest } \\
\hline Mean & & $78(13.85)$ \\
\hline Range & & $53-99$ \\
\hline \multicolumn{3}{|l|}{ Activities of Living Subtest } \\
\hline Mean & & $74(16.57)$ \\
\hline Range & & $42-110$ \\
\hline
\end{tabular}


was originally developed by Hilari, et al., to measure the quality of life of individuals with aphasia and was originally known as 'Stroke and Aphasia Quality of Life Scale39 (SAQOL-39) [18]. The Turkish adaption of Stroke and Aphasia Quality of Life Scale- 39 was made by Noyan-Erbaş and Toğram [30]. The SAQOL-39-TR has strong psychometric properties, with strong acceptability (minimal missing data (0-1.4); no floor/ceiling effects), test-retest reliability (ICC $=0.97$ ), internal consistency (Cronbach's $\alpha=0.70$ to 0.97 ), domain-total correlations ( $r=0.76$ to 0.85 ) and inter-domain correlations ( $r=0.40$ to 0.68$)$ [30]. After the application of SAQOL-39-TR, 5 different score types are obtained, in order to define the quality of life of participants: Physical (17 items, max score 85), communication (7 items, max score 35 ), psycho-social (11 items, max score 55) and energy (4 items, max score 20) subcategories and total score of the scale (39 items, max score 195). While higher scores represent a higher quality of life, lower scores represent the low quality of life $[18,30]$.

Caregiver Well-Being Scale was developed by Berg-Weger, et al. to measure the well- being of caregivers who were taking care of a family member with a chronic disease [31]. The Turkish adaptation, validity and reliability of the scale were done by Demirtepe and Bozo [32]. It aims to measure the daily operation levels of care givers in two categories: Basic Needs and Activities of Living. The maximum score is 110 in basic needs subtest and it has 22 items. Activity of life subtest includes 23 items with the maximum score of 115 . The total score of the scale is 225 [31,32].

\section{Procedure}

\section{Application of the scale}

The applications of the scales were conducted in an SLP research center in Turkey by the researcher. Before the application, the purpose and the importance of the Stroke and Aphasia Quality of Life-39 were explained to the individuals with aphasia and his/her proxy. SAQOL-39 TR scale was applied both to individuals with aphasia and their proxies individually and face to face by the researcher. Besides the SAQOL-39-TR scale, the Caregiver Well Being Scale was also applied to proxies. Two scale materials during the application to individuals with aphasia were used. One was the scale booklet which included general directives to be read by the individual with aphasia, sample items and scale items of subcategories. The other was the scoring table which would be used by the researcher. The scoring form of the researcher included the items of the scale, written directives of each item, and response types. Each item and their respective responses included in the scale were visually and aurally explained to individuals with aphasia. The latter were expected to visually follow the items in the booklet given to them as they are read by the researcher. The reason why the items in the scale were presented both visually and aurally was to facili- tate the comprehension of item contents for the individuals with aphasia. After the items and their responses were read by the researcher, individuals with aphasia were asked to point their choice with (for example: I did not have any difficulty). Those, who were able to express themselves asked to provide answers verbally. When the participants did not understand the item, the researcher gave an example and tried to clarify the meaning and the participants were given a chance for the response once again. During application, verbal explanations did not pass $30 \%$ of the total scale items. During the application, researcher repeated the phrase "during the last week" for each item. Shortest administration took 10 minutes and longest application took 45 minutes. Proxy of the individual with aphasia was given the Caregiver Well Being Scale and SAQOL-39 TR. Proxies were asked to fill out the forms by themselves.

\section{Data Analysis}

Statistical analysis was done with IBM SPSS Statistics for Windows, Version 21.0. Armonk, NY: IBM Corp. Released 2012. For descriptive statistics arithmetic mean \pm standard deviation method was used. Distribution of data obtained from the participants regarding the Stroke and Aphasia Life Quality-39 Scale scores were examined under Kolmogorov-Smirnov test and the data was normally distributed. Therefore, statistical analyses were done by using parametric tests. In order to determine the agreement between the individuals with aphasia and their proxies, Inter Correlation Coefficients (ICC) was calculated. ICC $<0.40$ signifies a poor agreement; ICC between $0.40-0.75$ means fair to good degree of agreement and ICC between 0.76-1.00 means excellent degree of agreement [33]. We applied $t$ tests analyses for two reasons, in order to examine the difference between the SAQOL-39 and subtest scores of proxies which are below and above the mean based on the Caregiver Well Being Scale scores and in order to determine the difference between their performances in the scale and subtests of individuals with aphasia and their proxies. We also calculated Pearson Correlation Coefficient to examine the relations of scale scores with variables and Caregiver Well Being scale.

\section{Results and Discussion}

\section{Results}

The agreement of level of individuals with aphasia and their proxies in SAQOL 39-TR scale: When we examined the Inter Correlation Coefficient-ICC, which we used to examine the agreement of reports of individuals with aphasia and their proxies in SAQOL 39-TR scale, we saw that there is excellent degree of agreement at scale level $(r=0.8)$, and fair to good degree of agreement at physical (0.7), communication (0.6), psycho-social (0.6) and energy (0.6) subtest levels.

Mean scores of individuals with aphasia and their proxies SAQOL-39-TR, mean difference between scores 
Table 2: Differences between the responses given by individuals with aphasia and by their proxies.

\begin{tabular}{|l|l|l|l|l|l|l|}
\hline SAQOL-39 TR & $\begin{array}{l}\text { IA } \\
\text { (mean (SD)) }\end{array}$ & $\begin{array}{l}\text { Proxy } \\
\text { (mean (SD)) }\end{array}$ & $\begin{array}{l}\text { Proxy-IA difference } \\
\text { (mean (SD)) }\end{array}$ & ICC & t & P \\
\hline Scale & $3.11(0.84)$ & $3.03(0.81)$ & $-0.08(0.54)$ & 0.8 & 0.38 & 0.706 \\
\hline Physical & $3.17(1.13)$ & $3.06(1.04)$ & $-0.10(0.83)$ & 0.7 & 0.39 & 0.702 \\
\hline Communication & $2.77(0.97)$ & $2.62(0.90)$ & $-0.15(0.83)$ & 0.6 & 0.65 & 0.521 \\
\hline Psycho-social & $3.27(0.85)$ & $3.35(0.84)$ & $-0.08(0.76)$ & 0.6 & -0.36 & 0.719 \\
\hline Energy & $2.92(1.08)$ & $2.66(1.15)$ & $-0.26(0.94)$ & 0.6 & 0.92 & 0.361 \\
\hline
\end{tabular}

SAQOL-39: Stroke and Aphasia Quality of life Scale-39; IA: Individual with Aphasia; ICC: Inter Correlation Coefficient.

Table 3: Correlation of age and gender related ADD auditory comprehension scores of individuals with aphasia with scale and its subtest scores.

\begin{tabular}{|l|l|l|l|l|l|l|l|l|l|l|l|}
\hline & \multicolumn{3}{l}{ Physical } & \multicolumn{3}{l|l}{ Communication } & \multicolumn{2}{l|}{ Psycho-social } & \multicolumn{2}{l|}{ Energy } & \multicolumn{2}{l|}{ Scale } \\
\hline & r & p & r & p & r & p & r & p & r & p \\
\hline Age & -0.21 & 0.24 & 0.04 & 0.83 & -0.06 & 0.75 & -0.27 & 0.14 & -0.17 & 0.36 \\
\hline Gender & -0.25 & 0.16 & -0.23 & 0.22 & -0.25 & 0.17 & -0.10 & 0.58 & -0.28 & 0.12 \\
\hline $\begin{array}{l}\text { ADD-Auditory } \\
\text { comprehension } \\
\text { score }\end{array}$ & 0.25 & 0.17 & $0.41^{*}$ & 0.02 & 0.21 & 0.26 & 0.21 & 0.26 & 0.32 & 0.08 \\
\hline
\end{tabular}

***means $p<0.001$, "means $p<0.005$, "means $p<0.05$.

Table 4: Correlation of SAQOL-39 scale scores of proxies of individuals with aphasia and caregiver well-being scale's basic needs and activities of living subtests scores.

\begin{tabular}{|c|c|c|c|c|c|c|c|c|c|c|}
\hline & \multicolumn{2}{|c|}{ Physical } & \multicolumn{2}{|c|}{ Communication } & \multicolumn{2}{|c|}{ Psycho-social } & \multicolumn{2}{|c|}{ Energy } & \multicolumn{2}{|c|}{ SAQOL-39 } \\
\hline & $\mathbf{r}$ & $\mathbf{p}$ & $\mathbf{r}$ & $\mathbf{p}$ & $\mathbf{r}$ & $\mathbf{p}$ & $\mathbf{r}$ & p & $\mathbf{r}$ & $\mathbf{P}$ \\
\hline $\mathrm{BN}$ & 0.234 & 0.198 & $0.411^{*}$ & 0.020 & $0.409^{*}$ & 0.020 & 0.008 & 0.965 & 0.338 & 0.058 \\
\hline VA & $0.395^{*}$ & 0.025 & $0.532^{* *}$ & 0.002 & $0.496^{* *}$ & 0.004 & 0.084 & 0.647 & $0.491^{* *}$ & 0.004 \\
\hline CGW & 0.340 & 0.057 & $0.504^{* *}$ & 0.003 & $0.483^{* *}$ & 0.005 & 0.052 & 0.776 & $0.446^{*}$ & 0.011 \\
\hline
\end{tabular}

BN: Basic Needs Subtest Scale; VA: Vital Activity Subtest Scale; CGW: Caregiver Well Being Scale; ***means $p<0.001 ;{ }^{* *}$ means $p<0.005$, "means $p<0.05$.

of proxies and individual with aphasia and agreement statistics are summarized below (Inter Correlation Coefficient-ICC, t value) (Table 2).

The correlation of SAQOL 39-TR scale scores of individuals with aphasia along with age, gender, ADD auditory comprehension scores: Table 3 indicates the correlation of SAQOL 39-TR scale and subtest scores of individuals with aphasia along with age, gender, ADD auditory comprehension scores.

We did not find any significant relation between SAQOL 39-TR scale and subtest scores (physical, communication, psycho-social, energy) of individuals with aphasia taking into account age, gender variables.

The correlation between SAQOL 39-TR scale scores of proxies of individuals with aphasia and Caregiver Well-Being Scale's Basic Needs and Activities of Living subtests scores: Table 4 shows the correlation between SAQOL 39-TR scale scores of proxies of individuals with aphasia and Caregiver Well-Being Scale's Basic Needs and Activities of Living subtests scores.

Based on their Caregiver Well-Being Scale's Basic Needs subtest scores of proxies, proxies were divided into two groups as above the mean (73) and below the mean, as with high scores $(n=22)$ and low scores ( $n=$ 10). We did not find any significant difference between the SAQOL-39-TR and subtest scores of proxies of individuals with aphasia.

Based on their Caregiver Well-being Scale's Activities of Living subtest scores of proxies, proxies were divided into two groups as above the mean (69) and below the mean, high scores $(n=18)$ and low scores $(n=14)$. We did not find any significant difference between the SAQOL-39-TR and subtest scores of proxies of individuals with aphasia. A significant difference between the groups with low and high scores within psycho-social subtest of SAQOL-39-TR scale for proxies of individuals with aphasia $(t=2.358, p<0.05)$ was found and the mean of this difference was appointed to the group with a higher mean. There was no significant difference between the groups with low and high scores within other subtests of SAQOL-39-TR scale.

\section{Discussion}

The purpose of the study is to examine the agreement between the reports given by individuals with 
aphasia and by their proxies to SAQOL-39 TR. In this study, differences and correlations between the perceptions of individuals with aphasia and their proxies on health-related quality of life were examined by examining the impacts of certain variables such as age, gender, Caregiver Well-Being Scale scores, which are all considered to affect the degree of consistency.

In the study, highest mean of subdomain of SAQOL39-TR was found in psycho-social (These means are 4.0, $3.52,3.27$ respectively). However, when the mean of SAQOL-39-TR and its subtest scores of individuals with aphasia is analyzed, we see that the highest figure is physical area in Hilari, et al. [23] and Noyan-Erbaş and Toğram's [30] studies. Physical subtest ranks second here while it comes first in other studies (3.17). While communication subdomain has the lowest mean in this study, the subdomain with lowest score in the studies of Hilari, et al. [23] and Noyan-Erbaş and Toğram [30] is energy. In total scale scores, the mean was 3.6 in Hilari, et al. [23], 3.36 in Noyan-Erbaş and Toğram [30] and 3.11 in this study.

We have also found that SAQOL-39-TR score difference is low $((-0.08)-(-0.26))$ at individuals with aphasia while the standard deviation of this mean is high (0.540.94). SAQOL-39-TR scores of individuals with aphasia and their proxies indicate strong agreement at overall scale level (0.8) and good level at physical (0.7), communication (0.6), psycho-social (0.6) and energy (0.6) sub domains. These results support the results of Hilari, et al. [23] and Ignatiou, et al. [24]. Hilari, et al., state that since SAQOL-39-TR was especially developed for measuring the quality of life of individuals with aphasia, this agreement turns out to be high. Contrary to the results of Hilari, Owen and Farelly [10] and Hilari, et al. [23], no significant difference was found between the SAQOL39-TR scale scores of individuals with aphasia and those of their proxies and that holds true for subtests as well (physical, communication, psycho-social, energy).

Regarding the relation of the SAQOL-39-TR scale and its subtests (physical, communication, psycho-social, energy) scores of individuals with aphasia with variables of age, auditory comprehension score, and gender, we have seen that there is a positively moving significant relation between auditory comprehension score and communication score $(r=0.414, p<0.05)$.

Auditory comprehension subtest score is one of the factors affecting the communication. Therefore, as the auditory comprehension scores of individuals with aphasia increase, the score of subtest communication also increases. Similarly, Noyan-Erbaş and Toğram reported significant correlation between the subtest auditory comprehension and the communication $(r=44$, $p<0.05$ ) [30]. It was observed that there was no significant relation between the scale and other variables. This may be due to limited sampling (with 32 individuals with aphasia) of the study as suggested by Ignatiou, et al. [28]. When the larger groups of participants included in the study, the impact of the variables may be more clearly analyzed. However, there was no relation between the age, gender and agreement as suggested by other studies as well [34,35].

When the correlation of scores of the SAQOL-39-TR scale and its subtests (physical, communication, psycho-social, energy) of proxies with age and gender variables is examined, no significant relation was found. We believe that this may also be due to limited sampling. One of the strong sides of this study is that proxies completely undertook the care of individuals with aphasia and they were spending most of their time with individuals with aphasia.

We examined the SAQOL-39-TR scores and Caregiver Well Being scores and saw that there was a positive significant relation between them $(r=0.446, p<0.05)$. We found the following significant relations: Between SAQOL-39-TR scale's scores and vital activity scores ( $r$ $=0.491, p<0.01$ ); between SAQOL-39-TR scale's physical subtest scores and vital activity scores $(r=0.395, p$ $<0.05$ ); between SAQOL-39-TR scale's communication subtest scores and vital activity scores $(r=0.532, p<$ $0.01)$, between basic necessities scores $(r=0.411, p<$ $0.05)$ and Caregiver Well Being Scale scores $(r=0.504$, $p<0.01$ ); between SAQOL-39-TR scale's psycho-social subtest scores and vital activity scores $(r=0.496, p<$ $0.01)$, between basic needs scores $(r=0.409, p<0.05)$ and Caregiver Well Being Scale scores $(r=0.483, p<$ 0.01).

Proxies were divided into groups based on the Caregiver Well Being Scale's basic needs subtest scores as high $(n=22)$ above the mean (73) and low $(n=10)$ below the mean. We did not find a significant difference between the SAQOL-39-TR total scale scores and those of subtests of proxies of individuals with aphasia. These results are in parallel to the results of some research $[23,27,28]$. However, it differs from results of a research [26], which suggests that psychological conditions of proxies of individuals with aphasia have impact on the agreement between the individual with aphasia and his/her proxy.

Proxies were divided into groups based on the Care Giver's Well Being Scale's vital activity subtest scores as high $(n=18)$ above the mean (69) and low $(n=14)$ below the mean. We found a significant difference between the low and high groups as per the SAQOL-39 TR scale's psycho-social subtest scores of proxies of individuals with aphasia $(t=2.358, p<0.05)$ and this difference was favoring the high defined group.

We did not find a significant difference between high and low groups in the SAQOL-39-TR overall scale and other subtest areas. As it was in the vital activity subtest, there was no significant difference between the high and low groups for the basic needs' subtest scale. There was only a significant difference between 
the high and low-scored groups in the psycho-social subtest score of SAQOL 39 scale. This means that as the life satisfaction of proxies of individuals with aphasia increases, they evaluate the psycho-social conditions of individuals with aphasia more positively.

\section{Conclusion and Suggestions}

The agreement between the individuals with aphasia and their proxies was examined based on their responses to the SAQOL-39-TR scale and it was excellent at scale level and fair to good at physical, communication, psycho-social, and energy. These results indicate that proxies of individuals with aphasia are a reliable source of information for evaluating the life qualities of individuals with aphasia. This information is beneficial for evaluating the life qualities of individuals with severe aphasia. As they have difficulty in understanding the questions, related to quality of life; defining their quality-of-life status and the progress after the stroke is also difficult. For this kind of conditions, proxies of them can answer the questions instead of the individuals with severe aphasia as there is an agreement with their responses.

The limitations of the study were related to the participants. The aphasia type was not homogeneous and the number of participants was not high enough.

In future studies, we suggest that further studies should target quality of life differences of individuals with different types of aphasia, impact of post-onset time after a stroke on life quality. It would also be better to study with larger sampling and by considering psychological conditions of individuals with aphasia, their socio-economical and educational status, in terms of variables such as relativity degree of proxies if they are working and to what degree they are educated.

\section{References}

1. World Health Organization (1997) Whoqol measuring quality of life. Geneva.

2. Eser E (2012) Health related quality of life. Celal Bayar Üniversitesi Tıp Fakültesi. Manisa.

3. Muus I, Petzold M, Ringsberg KC (2010) Health-related quality of life among Danish patients 3 and 12 months after TIA or mild stroke. Scand J Caring Sci 24: 211-218.

4. Kartsona A, Hilari K (2007) Quality of life in aphasia: Greek adaptation of the stroke and aphasia quality of life scale 39 item (SAQOL-39). Eura Medicophys 43: 27-35.

5. Pickard AS, Johnson JA, Feeny DH, Shuaib A, Carriere $\mathrm{KC}$, et al. (2004) Agreement between patient and proxy assessments of health-related quality of life after stroke using the EQ-5D and health utilities index. Stroke 35: 607-612.

6. Northcott S, Hilari K (2011) Why do people lose their friends after a stroke? Int J Lang Commun Disord 46: 524-534.

7. Carod-Artal J, Egido JA, González JL, Varela de Seijas E (2000) Quality of life among stroke survivors evaluated 1 year after stroke: Experience of a stroke unit. Stroke 31: 2995-3000.
8. Hallowell B, Chapey R (2008) Delivering language intervention services to adults with neurogenic communication disorders. In: R Chapey, Language Intervention Strategies in Aphasia and Related Neurogenic Communication Disorders. $\left(5^{\text {th }}\right.$ edn), Lippincott Williams \& Wilkins, Baltimore, 203-228.

9. Hilari K (2011) The impact of stroke: Are people with aphasia different to those without? Disabil Rehabil 33: 211-218.

10. Hilari K, Needle JJ, Harrison KL (2012) What are the important factors in health-related quality of life for people with aphasia? A systematic review. Arch Phys Med Rehabil 93: S86-S95.

11. Lee H, Lee $Y$, Choi H, Pyun SB (2015) Community integration and quality of life in aphasia after stroke. Yonsei Med J 56: 1694-1702.

12. Koleck M, Gana K, Lucot C, Darrigrand B, Mazaux JM, et al. (2017) Quality of life in aphasic patients 1 year after a first stroke. Qual Life Res 26: 45-54.

13. Bullier B, Cassoudesalle $H$, Villain $M$, Cogné $M$, Mollo $C$, et al. (2020) New factors that affect quality of life in patients with aphasia. Annals of Physical and Rehabilitation Medicine 63: 33-37.

14. Patrick DL, Erickson P (1993) Assessing health-related quality of life for clinical decision making. In: SR Walker, Quality of life assessment: Key issues in the 1990's. Kluwer Academic Publishers, Dordrecht, 11-63.

15. Mayou R, Bryant B (1993) Quality of life in cardiovascular disease. British Medical Journal 69: 460-466.

16. Royal College of Physicians (2008) National clinical guideline for stroke. ( $3^{\text {rd }}$ edn), Intercollegiate Stroke Working Party, RCP, London.

17. De Haan R, Aaronson N, Limburg M, Hewer RL, Van Crevel $H$ (1993) Measuring quality of life in stroke. Stroke 24: 320-327.

18. Hilari K, Byng S, Lamping DL, Smith SC (2003) Stroke and aphasia quality of life scale-39 (SAQOL-39): Evaluation of acceptability, reliability and validity. Stroke 34: 1944-1950.

19. Hilari K, Lamping DL, Smith SC, Northcott S, Lamb A, et al. (2009) Psychometric properties of the stroke and aphasia quality of life scale (SAQOL-39) in a generic stroke population. Clin Rehabil 23: 544-557.

20. Hilari K, Byng S (2009) Health-related quality of life in people with severe aphasia. Int $\mathrm{J}$ Lang Commun Disord 44: 193-205.

21. Cruice M, Worrall L, Hickson L, Murison R (2005) Measuring quality of life: Comparing family members' and friends' ratings with those of their aphasic partners. Aphasiology 19: 111-129.

22. Sneeuw KCA, Sprangers MAG, Aaronson NK (2002) The role of health care providers and significant others in evaluating the quality of life of patients with chronic disease. $J$ Clin Epidemiol 55: 1130-1143.

23. Hilari K, Owen S, Farrelly SJ (2007) Proxy and self-report agreement on the stroke and aphasia quality of life scale39. J Neurol Neurosurg Psychiatry 78: 1072-1075.

24. Ar Y, Karanci AN (2019) Turkish adult children as caregivers of parents with Alzheimer's disease: Perceptions and caregiving experiences. Dementia (London) 18: 882-902.

25. Karahan AY, Küçükşen S, Yilmaz H, Salli A, Güngör T, et al. (2014) Effects of rehabilitation services on anxiety, depression, care-giving burden and perceived social support 
of stroke caregivers. Acta Medica (Hradec Kralove) 57: 6872.

26. Williams LS, Bakas T, Brizendine E, Plue L, Tu W, et al. (2006) How valid are family proxy assessments of stroke patients' health-related quality of life? Stroke 37: 20812085.

27. Knapp P, Hewison J (1999) Disagreement in patient and carer assessment of functional abilities after stroke. Stroke 30: 934-938.

28. Ignatiou M, Christaki V, Chelas EN, Efstratiadou EA, Hilari K (2012) Agreement between people with aphasia and their proxies on health-related quality of life after stroke, using the Greek SAQOL-39g. Psychology 3: 686-690.

29. Maviş I, Toğram B (2009) Afazi Dil Değerlendirme Testi (ADD) manuel. Detay Yayinlari, İstanbul.
30. Noyan-Erbaş A, Toğram B (2016) Stroke and aphasia quality-of-life scale-39: Reliability and validity of the Turkish version. Int J Speech Lang Pathol 18: 432-438.

31. Berg-Weger M, Rubio DM, Tebb SS (2000) The caregiver well-being scale revisited. Health Soc Work 25: 255-263.

32. Demirtepe D, Bozo Ö (2009) The caregiver well-being scale validity and reliability study. Türk Psikoloji Yazilari 12: 28-37.

33. Rosner B (2000) Fundamentals of biostatistics. (6 $6^{\text {th }}$ edn), Pacific Grove, Duxbury, CA.

34. Choinière $M$, Melzack R, Girard N, Rondeau J, Paquin MJ (1990) Comparisons between patients' and nurses' assessment of pain and medication efficacy in severe burn injuries. Pain 40: 143-152.

35. McCusker J, Stoddard AM (1984) Use of a surrogate for the sickness impact profile. Med Care 22: 789-795. 